Erratum

\title{
The effect of the asymptotic response dynamics on the generalized synchronization
}

Shihong Wang a and Junzhong Yang

School of Science, Beijing University of Posts and Telecomunications, Beijing, 100876, P.R. China

Eur. Phys. J. B 55, 89 (2007)

Received 21 March 2007

Published online 4 April 2007 - (C) EDP Sciences, Società Italiana di Fisica, Springer-Verlag 2007

An error occurred in the second author's name of the article: Shihong Wang and Shihong Yang, Eur. Phys. J. B 55, 89 (2007). Instead of "Shihong Yang" you should read "Junzhong Yang". Therefore the correct reference of the article is:

The effect of the asymptotic response dynamics on the generalized synchronization

Shihong Wang and Junzhong Yang, Eur. Phys. J. B 55, 89 (2007).

a e-mail: shwang@bupt.edu.cn 